\title{
Motor neuron disease
}

\author{
M. Swash \\ Department of Neurology, Section of Neurological Sciences, The Royal London Hospital, London E1 1BB, \\ $U K$
}

\section{Introduction}

Motor neuron disease (MND) represents one of the major unsolved problems in neurology. The literature is characterized by numerous speculative theories, mostly derived by analogy with other recognized causes of motor neuron death such as poliomyelitis, or from toxic exposures such as lead poisoning. However, analogous reasoning of this type has not, so far, given insights into MND itself, or resulted in testable hypotheses. It is therefore relevant to consider the extent of established knowledge concerning the disease.

\section{Nomenclature}

In most countries other than those with a strong British cultural heritage motor neuron disease is known as amyotrophic lateral sclerosis (ALS) the name that $\mathrm{Charcot}^{1}$ used in 1869 to describe the typical pathology. In the United Kingdom the term MND has been preferred, used as a generic term to embrace all forms of acquired degenerative disease of the motor system, in which both upper and lower motor signs may be present. This is shown in Table I. Confusion in terminology often implies lack of understanding both of clinical definition and pathogenesis, and this is certainly so in MND. Most patients with MND show the clinical features of the ALS syndrome, only rare patients presenting with the clinical syndromes of primary lateral sclerosis, or progressive muscular atrophy. In about $10 \%$ of patients there is a syndrome of progressive bulbar palsy without other manifestations. However, in the terminal phase of the disease more widespread features are almost invariable. ${ }^{2}$

\section{The problem of research in MND}

Because MND is a progressive condition that affects the brain and spinal cord, there is no

Correspondence: M. Swash, M.D., F.R.C.P., M.R.C.Path.
Table I Classification of motor neuron diseases

A. Genetic disorders affecting anterior horn cells

e.g. spinal muscular atrophies hexosaminidase deficiency

B. Acquired disorders of the lower motor neuron

e.g. anterior poliomyelitis syringomyelia

C. Motor neuron disease syndromes

e.g. amyotrophic lateral sclerosis/motor neuron disease progressive muscular atrophy progressive lateral sclerosis progressive bulbar/pseudo-bulbar palsy

D. Rare motor neuron syndromes

e.g. Madras motor neuron disease motor neuron disease with deafness familial motor neuron disease familial spastic paraparesis ALS with frontal dementia

E. Diseases that may mimic motor neuron syndromes, but are not forms of MND

e.g. motor neuropathy with conduction block radiation myelopathy heavy metal poisoning amyotrophic form of Creutzfeldt-Jakob disease

opportunity to study the biology of the affected parts of the motor system during life. Histopathological research in living patients is therefore necessarily restricted to studies of muscle and its innervation. The motor system in the brain and spinal cord is available for study only after death, when the disease has reached its maximum extent, and with the inevitable problems of post-mortem artefact. Little is therefore known about the process of motor neuronal death in the spinal cord or in the somatic brain stem nuclei, and its relation to involvement of the upper motor neuron, apart 
from what has been learnt from clinical and physiological studies during life. The absence of an animal model of the disease has further restricted research. Since the disease, as far as is known, has no systemic effect, there is no abnormality in blood or other tissues that could be investigated in parallel to the involvement of the nervous system. Imaging of the nervous system by computerized tomographic (CT) scanning reveals mild frontal and parietal atrophy, ${ }^{3}$ but magnetic resonance imaging (MRI) studies can demonstrate degeneration taking place in the upper motor neuron pathway in the brain and cerebral peduncles in some cases.

\section{Clinical clues}

The clinical features of the disease themselves indicate the framework that any formulation of causation must seek to explain. The variability in presentation with upper or motor neuron features predominating, the presentation in some cases, particularly in middle-aged women, with bulbar features, and the gradual progression to more generalized disease with the expression of the complete syndrome of amyotrophic lateral sclerosis, is rather characteristic. It has been considered that these progressive features imply primary involvement of anterior horn cells or, conversely, that the disease is primarily expressed in the upper motor neuron, involvement of the lower motor neuron developing as a secondary feature. These interpretations of the pathogenesis and spread of the disease are examples of the essentially speculative reasoning alluded to above.

Fatiguability, cramps and weakness are all symptoms of neurogenic disease that are common in the course of MND and that do not require specific explanation in MND as distinct from their occurrence in other neurogenic disorders. The early neurogenic features, however, are frequently asymmetrical although, even at this time, there are usually abnormalities, such as fasciculation, that are detectable in other muscle groups and that indicate more widespread involvement of the motor system. Two-thirds of patients present with weakness of one limb, and this is slightly more likely to consist of weakness of a leg rather than of an arm. The distribution of initial involvement and the subsequent development of more widespread involvement does not follow any predictable pattern, and the factors leading to the specific affection of certain muscle groups at the onset in any individual case are unknown. There is no evidence that the pattern of use of a group of muscles is relevant to the clinical progression of the disease, although this is an attractive notion. However, electromyogram studies suggest that the disease is widespread from the earliest point of diagnosis, when there is already atrophy of some muscle groups. ${ }^{4,5}$ Retrospective studies of clinical data sometimes indicate that the disease may begin several years before presentation with muscular weakness or wasting; thus there may be a long preclinical phase. ${ }^{6}$ There is some controversy as to whether progression of the disease is linear ${ }^{7}$ or non-linear. Studies of the batting averages of Lou Gehrig, the celebrated American professional baseball player who developed MND during his playing career, suggest that there was an abrupt fall-off in performance about 2 years before his death. ${ }^{8}$ Brooks and his colleagues have studied the natural history of MND in some detail in relation to the possible design of clinical trials of putative new treatments, and have found a linear early phase, followed by a slowing in the rate of progression in the later stages of the disease. ${ }^{9}$ Assessment of these data requires recognition of the necessity for up to $80 \%$ loss of motor neurons before clinical weakness develops, ${ }^{10}$ because of the superimposed factor of compensatory reinnervation.

The occurrence of sensory symptoms in MND is common, although objective sensory abnormalities are rarely, if ever, found. ${ }^{11}$ The sensory threshold to quantitative testing may be reduced, ${ }^{12,13}$ especially the thermal threshold. ${ }^{14}$ These findings have bee interpreted in terms of involvement of smat unmyelinated sensory axons in the peripheral nefo vous system in the disease, and are perhaps also consistent with the finding of pallor of the medial parts of the posterior columns in the cervical cord in autopsy studies.

Autonomic function is generally considered normal in MND, although there is evidence of abnormalities in cardiovascular tests, ${ }^{15}$ a finding that is consistent with the slight loss of neurons in the intermediolateral cell columns observed by Kennedy and Duchen. ${ }^{16}$

Dementia is a recognized feature of the later stages of the disease, but its frequency is difficult to define because of the problems in assessment of mental state in severely ill patients. It may occur in about $15 \%$ of cases. ${ }^{17}$ Neary and colleagues described a specific syndrome of frontal lobe dementia with MND, in which there was vacuolar change in frontal and temporal lobes. ${ }^{18}$ The clinical and pathological features of the dementia that sometimes occurs in MND are not those of Alzheimer's disease.

Spinocerebellar neurons are also involved in the disease. ${ }^{19,20}$ These findings outside the motor system clearly indicate that MND is a disorder that affects the central and peripheral nervous systems more widely than has previously been recognized Any concept of causation must seek to encompass these features.

The relative invulnerability of certain groups of 
motor neurons to the disease process has excited considerable attention. Patients with MND usually retain eye movement, do not suffer from stridor, and do not become incontinent since the muscles responsible for these functions are relatively resistant to neurogenic atrophy in the disease. The external ocular movements often show abnormalities of slow pursuit in the later stages of the disease,${ }^{21}$ and partial external ophthalmoplegia has been recognized in a few cases with long survival $^{22,23}$ with loss of neurons in the ocular motor nuclei. The Onuf nucleus that supplies the external anal and urinary sphincter muscles of the pelvic floor is located in the S2 and S3 segments of the spinal cord. These neurons are uninvolved in MND at autopsy, ${ }^{24}$ and they are also spared in acute poliomyelitis. ${ }^{25}$ No satisfactory explanation for these findings has been adduced, but it is clear that the synaptic anatomy of the Onuf neurons, in particular, differs from that of other motoneuronal groups in the anterior horn of the spinal cord, and that these neurons are in close functional contact with autonomic neurons innervating the involuntary smooth sphincter musculature. ${ }^{26}$ The biological mechanisms accounting for the relative resistance of these neurons to the disease process are clearly of some interest in relation to the pathogenesis of the disease.

\section{Clinical variance}

The different clinical syndromes that occur in the disease appear to offer no clues to understanding pathogenesis, but rather to indicate differing degrees of vulnerability of the different parts of the motor system in the disease. However, certain clinical variants that occur in certain countries have been much studied. For example, the Guamanian form of the disease (Western Pacific ALS) is a form of the disease occurring with a frequency about 100 times that of the spontaneous disease in Western countries. It has been suggested that this form of MND is due to dietary ingestion of cycads, or to the dietary deficiency of calcium and magnesium that is endemic on Guam, but these concepts have remained speculative and it is significant that no model of the disease produced by these factors has been produced. ${ }^{27} \mathrm{~A}$ predominantly distal form of the disease, usually confined to one upper or lower limb has been recognized in Japan, Sri Lanka and India, and in a lesser frequency, in Europe. ${ }^{28}$ This is currently regarded as an extreme example of resistance to the progressive dissemination of the disease that is so characteristic of most sporadic cases.

Genetic factors also have to be considered. The disease is of late onset, with a peak incidence in the sixth decade. As in all diseases of late onset the ascertainment of familial cases is likely to be inaccurate. The example of the spinal muscular atrophies, which are inherited as autosomal recessive traits, and known to be associated with a genetic locus on chromosome $5 \mathrm{q} 11.2-13.3^{29}$ is important since this also is a disorder in which there is progressive motor neuronal death, although without associated degeneration of the upper motor neuron, and without the mild, but widespread involvement of other neuronal systems in the central nervous system. Could the $10 \%$ familial concurrence of MND noted in almost all series of the disease represent under-ascertainment of a genetic predisposition? This question is likely to be resolved only with the discovery of the locus of the genetic abnormality underlying familial disease, and requires study of informative families. Unfortunately, most families in which a familial occurrence of the disease is recognized are uninformative since few, if any, family members are surviving at the time of ascertainment.

\section{Other current areas of research}

There are several strands of ideas underlying current research into MND. These relate to the notion that exogenous excitotoxins may play a role in the process of selective neuronal death that characterizes the disease, that mineral deficiency may be important, that the disease may in some way be related to latent infection with polio virus, that autoimmunity may play a role, and that studies of the molecular pathology of motor neurons in MND will yield insights into the disturbance of molecular biology that must underlie neuronal death in the disease. All these avenues are actively being pursued, but only the latter is directly related to MND itself.

The molecular pathology approach is currently interesting because of the emphasis that has been placed on unravelling the pathology and biochemistry of the characteristic intra-neuronal inclusions found in remaining motor neurons at autopsy. Using antibodies to heat shock proteins it has been shown that these remaining motor neurons contain ubiquitinated blob-like and skeinlike cytoplasmic inclusions. ${ }^{30,31}$ These inclusions are found also in the proximal axons of the ventral horn grey matter and to a lesser extent in the upper motor neuron. Lewy-like inclusions, that are also found in motor neurons in the disease are associated with ubiquitination of the $15 \mathrm{~nm}$ filaments that constitute this inclusion. ${ }^{32}$ Accumulations of phosphorylated neurofilaments are commonly found in familial MND, but are less characteristic of the sporadic disorder. ${ }^{33,34}$ Bunina bodies, also found in anterior horn cells, are rarely associated with ubiquitin, and other heat shock 
proteins, for example the $72 \mathrm{kD}$ heat shock proteins are not associated with ubiquitination. ${ }^{35}$ The question naturally arises as to the significance of these findings. Unfortunately it is probable that the ubiquitination response is non-specific, representing activation of a common pathway for cytoplasmic degradation of damaged or inactivated cellular proteins, rather than a fundamental part of the cellular pathology of MND. Nonetheless, this ubiquitinated inclusion response in damaged anterior horn cells appears to be a specific and almost diagnostic finding in MND, and as such must represent an important cellular response. It is possible that it is induced by damage to the cytoskeletal framework of the cell, perhaps to abnormalities of the microtubular transport proteins, and much current work is directed to this concept.

One of the major difficulties in trying to bring together the strands of current work on MND is that much of this work is disparate. For example, there has recently been a renewal of interest in the old idea that MND might represent a late complication, or a late sequel to remote infection with poliovirus, either as a latent infection or by some other, undefined mechanism. ${ }^{36}$ The evidence for this concept is largely epidemiological, and relates to the apparent increase in the incidence of the disease that has been reported from surveys in several countries. ${ }^{37,38}$ Extensive studies designed to demonstrate poliovirus antigen or antibody in the tissues of patients with MND have been consistently negative, and it therefore seems difficult to reconcile the epidemiological evidence with the other findings. Resolution of these problems awaits more definitive data from the developing molecular biological approaches to the disease.

\section{References}

1. Charcot, J.M. Lectures on the Diseases of the Nervous System. Lecture XIII, 2nd series. New Sydenham Society, London, 1881 (republished 1962), Hafner, New York, p. 192.

2. Caroscio, J.T., Calhuon, W.F. \& Yahr, M.D. Prognostic factors in motor neuron disease; a prospective study of longevity. In: Rose, F.C. (ed) Research Progress in Motor Neurone Disease. Pitman, London, 1984, pp. 34-43.

3. Polari, M., Marscherpa, C., Faggi, L. et al. Cerebral atrophy in motor neuron disease evaluated by computed tomography. J Neurol Neurosurg Psychiat 1982, 45: 1102-1105.

4. Swash, M. \& Schwartz, M.S. A longitudinal study of changes in motor units in motor neuron disease. J Neurol Sci 1982, 56: 185-197.

5. Schwartz, M.S. \& Swash, M. Pattern of involvement in the cervical segments in the early stages of motor neuron disease; a single fibre EMG study. Acta Neurol Scand 1982, 65: 424-431.

6. Swash, M. \& Ingram, D.A. Preclinical and subclinical events in motor neuron disease. J Neurol Neurosurg Psychiat 1988, 51: $165-168$.

7. Munsat, T.L., Andres, P.L., Finison, L. et al. The natural history of motor neuron loss in amyotrophic lateral sclerosis. Neurology 1988, 38: 937-941.

\section{Conclusions}

Understanding of the pathogenesis of MND is currently very much a matter for speculation. The $\bigcirc$ available data do not allow formulation of testable $c$ hypotheses, and consist mainly of a number of chance observations. Definition of the disease, however, may be achieved from molecular biology, which is beginning to have an impact on our understanding of the molecular pathology of the neuronal cytoskeleton. The new information strongly suggests that there are relatively specific abnormalities in the cytoskeleton of motor neurons in the disease. So far, there is no experimental model of the disease, either occurring naturally or in response to the administration of exogenous toxins. It is possible that further exploration of the neurochemistry of neurotoxic substances may provide an insight into the mechanisms of neuronal death in this late onset neuronal degeneration. Studies of the pathogenesis of cytoplasmic inclusions in this and other degenerative diseases promise to be important in this context.

In many ways MND has been the 'Cinderella' of the degenerative disorders, receiving little attention from researchers involved in disorders with a longer life expectancy such as Parkinson's disease or muscular dystrophy. In addition there has bee little ear-marked funding available to encourage investigators to invest in the study of this disordes The formation of a world-wide network of patientled groups such as the Motor Neurone Disease Association in the United Kingdom, has done much to remedy this situation and it is profoundly to be hoped that this will result in greater understanding and eventually a means of prevention of this tragic disease.

8. Kasarkis, E.J. \& Winslow, N. When did Lou Gehrig' personal illness begin? Neurology 1989, 39: 1243-1245.

9. Brooks, B.R., Sufit, R.L., Clough, J.A. et al. Isokinetic and functional evaluation of muscle strength over time in amyotrophic lateral sclerosis. In: Munsat, T.L. (ed) Quantification of Neurologic Deficit. Boston, Butterworths, 1989, pp. 143154.

10. Sharrard, W.J.W. The distribution of permanent paralysis in the lower limb in poliomyelitis. J Bone Joint Surg 1955, 37B: 540-548.

11. Mulder, D.W. Clinical limits of amyotrophic lateral sclerosis. In: Rowland, L.P. (ed) Human Motor Neuron Diseases. New York, Raven Press, 1982, pp. 15-29.

12. Shahani, B., Davies-Jones, G.A.B. \& Russell, W.R. Motor neuron disease; further evidence for an abnormality of disease metabolism. J Neurol Neurosurg Psychiat 1971, 34: 185- 191 .

13. Mulder, D.W., Bushek, W., Spring, E. et al. Motor neuron disease/ALS; evaluation of detection thresholds in cutaneous sensation. Neurology 1983, 33: 1625-1627. 
14. Jamal, G.A., Weir, A.I., Hansen, S. \& Ballantyne, J.P. Sensory involvement in motor neuron disease; further evidence from automated thermal threshold determination. $J$ Neurol Neurosurg Psychiat 1985, 48: 906-910.

15. Chida, K., Sakamaki, S. \& Takasu, T. Alteration in autonomic function and cardiovascular regulation in amyotrophic lateral sclerosis. $J$ Neurol 1989, 236: 127-130.

16. Kennedy, P.G.E. \& Duchen, L.W. A quantitative study of intermediolateral column cells in motor neuron disease and the Shy-Drager syndrome. J Neurol Neurosurg Psychiat 1985, 48: 1103-1106.

17. Hudson, A.J. Amyotrophic lateral sclerosis and its association with dementia in Parkinsonism and other neurological disorders a review. Brain 1981, 104: 217-247.

18. Neary, D., Snowden, J.S., Mann, D.M.A., Northern, B., Goulding, P.J. \& MacDermott, N. Frontal lobe dementia and motor neuron disease. J Neurol Neurosurg Psychiat 1990, 53: 23-32.

19. Swash, M., Scholtz, C.L., Vowles, G. \& Ingram, D.A. Selective and asymmetric degeneration of corticospinal and spinocerebellar tracts in motor neuron disease. $J$ Neurol Neurosurg Psychiat 1988, 51: 785-789.

20. Williams, C., Kozlowski, M.A., Hinton, D.R. \& Miller, C.A. Degeneration of spinocerebellar neurons in amyotrophic lateral sclerosis. Ann Neurol 1990, 27: 215-225.

21. Jacobs, L., Bozian, D., Heffner, R.R., Jr et al. An eye movement disorder in amyotrophic lateral sclerosis. Neurology 1981, 31: 1282-1287.

22. Harvey, D.G., Torack, R.M. \& Rosenbaum, H.E. Amyotrophic lateral sclerosis with ophthalmoplegia: a clinicopathologic study. Arch Neurol 1979, 36: 615-617.

23. Hayashi, H. \& Kato, S. Total manifestations of amyotrophic lateral sclerosis (ALS) in the totally locked-in state. $J$ Neurol Sci 1989, 93: 19-35.

24. Mannen, T., Iwata, M., Toyokura, Y. et al. Preservation of a certain motor neuron group of the sacral cord in amyotrophic lateral sclerosis; its clinical significance. $J$ Neurol Neurosurg Psychiat 1977, 40: 464-469.

25. Kohima, H., Furuta, Y., Fujita, M. et al. Onuf's nucleus is resistant to poliovirus. J Neurol Sci 1989, 93: 85-92.

26. De Groat, W.C. Central neural control of the lower urinary tract. In: Bock, G. \& Whelan, J. (eds) Neurobiology of Incontinence. CIBA Foundation Symposium 151. Chichester, John Wiley, 1990, pp. 27-56.
27. Garrato, R.M., Swyt, C., Fiori, C.E., Yanagihara, R. \& Gajdusek, D.C. Intraneuronal deposition of calcium and aluminium in amyotrophic lateral sclerosis of Guan. Lancet 1985, ii: 1353.

28. Chaine, P., Bouche, P., Leger, J.M. et al. Atrophie musculaire progressive localisée à la main. Rev Neurol 1988, 144: $759-763$.

29. Brzustowicz, L.M., Lehner, T., Castilla, L.H. et al. Genetic mapping of chronic childhood-onset spinal muscular atrophy to chromosome 5q11.2-13.3. Nature 1990, 344: 540-541.

30. Leigh, P.N., Anderton, B.H., Dodson, A. et al. Ubiquitin deposits in anterior horn cells in motor neuron disease. Neurosci Lett 1988, 93: 197-203.

31. Lowe, J., Lennox, G., Jefferson, D. et al. A filamentous inclusion body within anterior horn neurones in motor neurone disease defined by immunocytochemical localisation of ubiquitin-protein conjugates. Neurosci Lett 1988, 93: 203-210.

32. Murayama, S., Akawa, Y., Mori, H. et al. Immunocytochemical and ultrastructural studies of Lewy body-like hyaline inclusions in familial amyotrophic lateral sclerosis. Acta Neuropath 1989, 78: 143-152.

33. Mizusawa, H., Matsumoto, S., Yen, S.-H. et al. Focal accumulation of phosphorylated neurofilaments within anterior horn cells in familial amyotrophic lateral sclerosis. Acta Neuropath 1989, 79: 37-43.

34. Leigh, P.N., Dodson, A., Swash, M. et al. Cytoskeletal abnormalities in motor neuron disease; an immunocytochemical study. Brain 1989, 112: 521-535.

35. Garofalo, O., Kennedy, P.G.E., Swash, M. et al. Ubiquitin and heat shock protein expression in amyotrophic lateral sclerosis. Neuropath Appl Neurobiol 1991, 17: 39-46.

36. Salman, L.A. \& Riley, H.A. The relation between chronic anterior poliomyelitis or progressive spinal muscular atrophy and an antecedent attack of acute anterior poliomyelitis. Bull Neurol Inst NY 1935, 4: 35-63.

37. Martyn, C.N., Barker, D.J.P. \& Osmond, C. Motor neuron disease and past poliomyelitis in England and Wales. Lancet 1988, 1: 1319-1322.

38. Durrleman, S. \& Alperovitch, A. Increasing trend of ALS in France and elsewhere; are the changes real? Neurology 1989, 39: 768-773. 